\title{
Neutrino physics from cosmological observations
}

\author{
Steen Hannestad ${ }^{\mathrm{a}}$

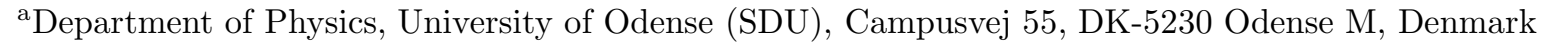

We review the current status of neutrino cosmology, focusing mainly on the question of the absolute values of neutrino masses and the possibility of a cosmological neutrino lepton asymmetry.

\section{Neutrino masses}

The absolute value of neutrino masses are very difficult to measure experimentally. On the other hand, mass differences between neutrino mass eigenstates, $\left(m_{1}, m_{2}, m_{3}\right)$, can be measured in neutrino oscillation experiments. Observations of atmospheric neutrinos suggest a squared mass difference of $\delta m^{2} \simeq 3 \times 10^{-3} \mathrm{eV}^{2}$ [1, 2]. While there are still several viable solutions to the solar neutrino problem the so-called large mixing angle solution gives by far the best fit with $\delta m^{2} \simeq 5 \times 10^{-5}$ $\mathrm{eV}^{2}$ [3, 1 . (see also contributions by A. Hallin and A. Smirnov in the present volume).

In the simplest case where neutrino masses are hierarchical these results suggest that $m_{1} \sim 0$, $m_{2} \sim \delta m_{\text {solar }}$, and $m_{3} \sim \delta m_{\text {atmospheric. If the hi- }}$ erarchy is inverted [5 10] one instead finds $m_{3} \sim$ $0, m_{2} \sim \delta m_{\text {atmospheric }}$, and $m_{1} \sim \delta m_{\text {atmospheric }}$. However, it is also possible that neutrino masses are degenerate [11 21], $m_{1} \sim m_{2} \sim m_{3} \gg$ $\delta m_{\text {atmospheric }}$, in which case oscillation experiments are not useful for determining the absolute mass scale.

Experiments which rely on kinematical effects of the neutrino mass offer the strongest probe of this overall mass scale. Tritium decay measurements have been able to put an upper limit on the electron neutrino mass of $2.2 \mathrm{eV}$ (95\% conf.) 22 (see also the contribution by Ch. Weinheimer in the present volume). However, cosmology at present yields an even stronger limit which is also based on the kinematics of neutrino mass.

Neutrinos decouple at a temperature of 1$2 \mathrm{MeV}$ in the early universe, shortly before electron-positron annihilation. Therefore their temperature is lower than the photon temperature by a factor $(4 / 11)^{1 / 3}$. This again means that the total neutrino number density is related to the photon number density by

$n_{\nu}=\frac{9}{11} n_{\gamma}$

Massive neutrinos with masses $m \gg T_{0} \sim$ $2.4 \times 10^{-4} \mathrm{eV}$ are non-relativistic at present and therefore contribute to the cosmological matter density 23 25]

$\Omega_{\nu} h^{2}=\frac{\sum m_{\nu}}{92.5 \mathrm{eV}}$,

calculated for a present day photon temperature $T_{0}=2.728 \mathrm{~K}$. Here, $\sum m_{\nu}=m_{1}+m_{2}+m_{3}$. However, because they are so light these neutrinos free stream on a scale of roughly $k \simeq$ $0.03 m_{\mathrm{eV}} \Omega_{m}^{1 / 2} h \mathrm{Mpc}^{-1}$ [26 28]. Below this scale neutrino perturbations are completely erased and therefore the matter power spectrum is suppressed, roughly by $\Delta P / P \sim-8 \Omega_{\nu} / \Omega_{m}$ [28].

This power spectrum suppression allows for a determination of the neutrino mass from measurements of the matter power spectrum on large scales. This matter spectrum is related to the galaxy correlation spectrum measured in large scale structure (LSS) surveys via the bias parameter, $b^{2} \equiv P_{g}(k) / P_{m}(k)$. Such analyses have been performed several times before [29,30, most recently using data from the $2 \mathrm{dF}$ galaxy survey [31]. This investigation finds an upper limit of 1.8-2.2 $\mathrm{eV}$ for the sum of neutrino masses. However, this result is based on a relatively limited cosmological parameter space. For the same data and an even more restricted parameter space an upper limit of $1.5 \mathrm{eV}$ was found [32]. 
It should also be noted that, although massive neutrinos have little impact on the cosmic microwave background (CMB) power spectrum, it is still necessary to include CMB data in any analysis in order to determine other cosmological parameters.

When calculating bounds on the neutrino mass from cosmological observations great care must be taken, because if the analysis is based on a too restricted parameter space, possible parameter degeneracies cannot be studied and the bound on $m_{\nu}$ can become artificially strong.

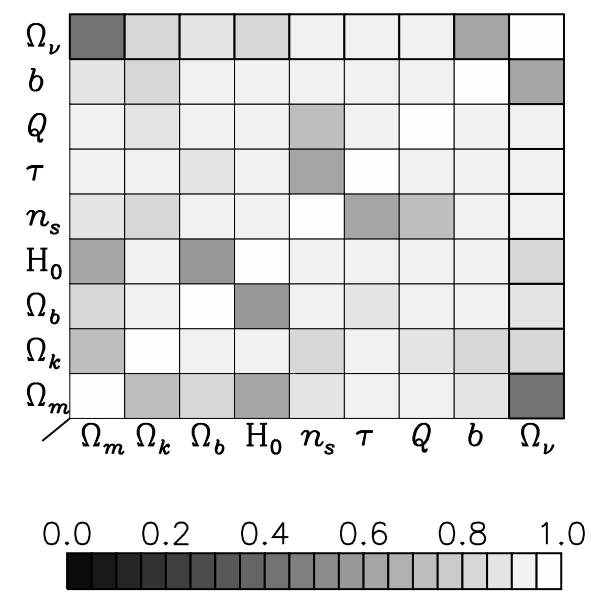

Figure 1. Values of the parameter $r_{i j}$, defined in Eq. (3).

In Fig. 1 the degeneracy between different cosmological parameters is shown in form of the quantity

$r_{i j}=\frac{\sigma_{j \text { fixed }}\left(\theta_{i}\right)}{\sigma\left(\theta_{i}\right)} \leq 1$,

i.e. the decrease in uncertainty of a measurement of parameter $i$ when parameter $j$ is fixed. From this figure it can be seen that there are significant degeneracies between $\Sigma m_{\nu}$ and other parameters, most notably $b$, the bias parameter, and $\Omega_{m}$, the matter density.
In Ref. [33] a full numerical likelihood analysis was performed using a slightly restricted parameter space with the following free parameters: $\Omega_{m}$, $\Omega_{b}, H_{0}, n_{s}, Q, b$, and $\tau$. The analysis was further restricted to flat models, $\Omega_{k}=0$. This has very little effect on the analysis because there is little degeneracy between $m_{\nu}$ and $\Omega_{k}$. In order to study the effect of the different priors three different cases were calculated, the priors for which can be seen in Table I. The BBN prior on $\Omega_{b} h^{2}$ comes from Ref. [34]. The actual marginalization over parameters other than $\Omega_{\nu} h^{2}$ was performed using a simulated annealing procedure [35].

Fig. 2 shows $\chi^{2}$ for the three different cases as a function of the $m_{\nu}$. The best fit $\chi^{2}$ values are $24.81,25.66$, and 25.71 for the three different priors respectively. In comparison the number of degrees of freedom are 34,35 , and 36 , meaning that the fits are compatible with expectations, roughly within the $68 \%$ confidence interval.

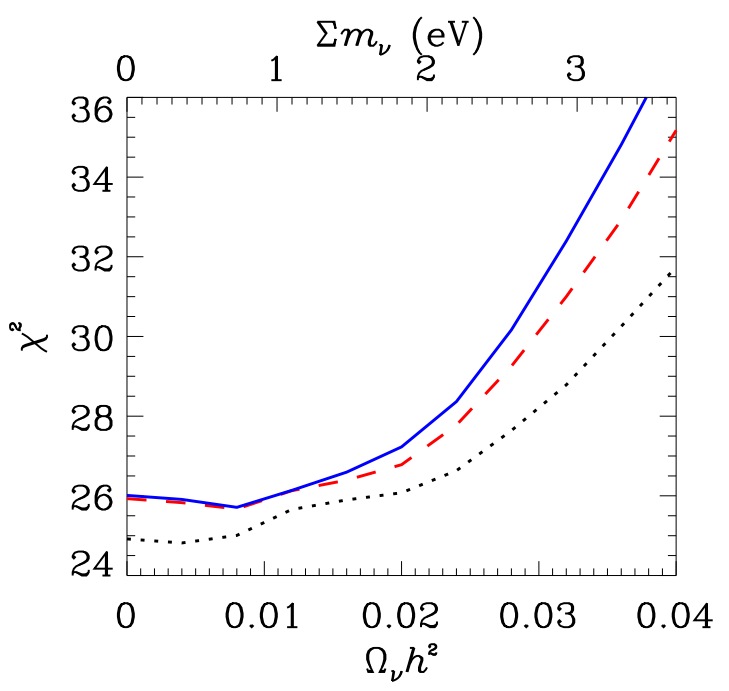

Figure 2. $\chi^{2}$ as a function of $\Omega_{\nu} h^{2}$, plotted for the three different priors. The dotted curve is for CMB+LSS, the dashed for $\mathrm{CMB}+\mathrm{LSS}+\mathrm{BBN}+H_{0}$, and the full curve for $\mathrm{CMB}+\mathrm{LSS}+\mathrm{BBN}+H_{0}+\mathrm{SNIa}$.

The $95 \%$ confidence limit on $m_{\nu}$ was identified 
with the point where $\Delta \chi^{2}=4$. These limits are shown in Table II. For the most restrictive prior we find a $95 \%$ confidence upper limit of $\sum m_{\nu} \leq$ $2.47 \mathrm{eV}$. This is compatible with the findings of Ref. [31] who derived that $\sum m_{\nu} \lesssim 1.8-2.2 \mathrm{eV}$ for a slightly more restrictive parameter space.

Based on the present analysis we consider $\sum m_{\nu} \leq 3 \mathrm{eV}$ (95\% conf.) a robust upper limit on the sum of the neutrino masses. This corresponds roughly to the value found for the CMB+LSS data alone without any additional priors. Even though this value is significantly higher than what is quoted in Ref. [31], it is still much more restrictive than the value $\sum m_{\nu} \leq 4.4 \mathrm{eV}$ [36] found from $\mathrm{CMB}$ and $\mathrm{PSCz}$ [37] data. As is also discussed in Ref. 31 the main reason for the improvement is the much greater precision of the $2 \mathrm{dF}$ survey, compared to the $\mathrm{PSCz}$ data [37].

\section{Cosmological neutrino lepton asymme- try}

Cosmological observations, in addition to the information they yield on neutrino masses, also provide a powerful means of determining the presence of a non-zero cosmological neutrino lepton number or additional sterile neutrino species.

In addition to affecting structure formation neutrinos also contribute relativistic energy density in the early universe. This has a profound effect on big bang nucleosynthesis as well as the CMB formation. The Friedmann equation, $H^{2}=8 \pi G \rho / 3$, yields a relationship between temperature and expansion rate. The beta reactions which maintain equilibrium between neutrons and protons in the early universe freeze out roughly when $\Gamma / H \sim 1$. If relativistic energy density is added then $H$ is larger for a given temperature and the beta reactions freeze out faster. The neutron to proton ratio is in equilibrium given by $n / p \propto e^{-Q / T}$, with $Q=m_{n}-m_{p}=1.293 \mathrm{MeV}$, so that if relativistic energy density is added more neutrons survive. This in turn means that more helium is formed, the dependence being roughly $Y_{P} \sim N_{\nu}^{1 / 6}$, and indeed observations of the primordial helium abundance can be used to constrain the amount of relativistic energy density.

The standard way to parameterise such en- ergy density is in equivalent number of neutrino species $N_{\nu} \equiv \rho_{R} / \rho_{\nu, 0}$, where $\rho_{\nu, 0}$ is the energy density of a standard neutrino species. At present the bound is roughly $N_{\nu} \lesssim 3.5-4$ [38.39].

At first sight this bound can be translated directly into a bound on the neutrino lepton number as well, because a non-zero lepton number yields additional energy density.

$N_{\nu}=3+\frac{30}{7}\left(\frac{\mu}{\pi T}\right)^{2}+\frac{15}{7}\left(\frac{\mu}{\pi T}\right)^{4}$,

assuming that only one neutrino species has nonzero lepton number.

However, there is a fundamental difference in that the bound is flavour sensitive. The electron flavour neutrinos enter directly into the beta reactions, and an electron neutrino lepton number therefore has a different influence on BBN than muon or tau neutrino lepton numbers 40].

In practise this means that a large positive chemical potential in muon and tau neutrinos can be compensated by a small electron neutrino chemical potential. Of course such models are quite contrived, but it is highly desirable with independent methods for determining the cosmological neutrino lepton numbers.

It turns out that the CMB is at least in principle also an excellent probe of the relativistic energy density. The reason is that an increase in the relativistic energy density delays matter radiation equality, which in turn leads to an increase of the so-called early integrated Sachs-Wolfe (ISW) effect. In the power spectrum this shows up as an increase in power around the scale of the particle horizon slightly after recombination, i.e around the scale of the first acoustic peak. This effect can be seen in Fig. 3.

This effect has been used previously to constrain $N_{\nu}$ using data from the best present day experiments 41 44, namely Boomerang 445], Maxima [46], CBI [47] and DASI [48]. Unfortunately the data is not yet of sufficient accuracy to yield constraints anywhere near as strong as BBN. However, on the other hand they do not suffer from the same problems of being flavour sensitive. It is therefore possible to combine BBN and CMB constraints to yield a non-trivial bound on neutrino lepton chemical potentials 499. From a 
Table 1

The different priors on parameters other than $\Omega_{\nu} h^{2}$ used in the analysis of Ref. [33].

\begin{tabular}{lccc}
\hline Parameter & CMB + LSS & $\begin{array}{c}\text { CMB + LSS } \\
+ \text { BBN }+H_{0}\end{array}$ & $\begin{array}{c}\text { CMB + LSS + BBN } \\
+ \text { BBN }+H_{0}+\text { SNIa }\end{array}$ \\
\hline$\Omega_{m}$ & & $0.1-1$ & $0.28 \pm 0.14$ \\
$\Omega_{b} h^{2}$ & $0.1-1$ & $0.020 \pm 0.002$ & $0.020 \pm 0.002$ \\
$h$ & $0.008-0.040$ & $0.70 \pm 0.07$ & $0.70 \pm 0.07$ \\
$n$ & $0.4-1.0$ & $0.66-1.34$ & $0.66-1.34$ \\
$\tau$ & $0.66-1.34$ & $0-1$ & $0-1$ \\
$Q$ & $0-1$ & free & free \\
$b$ & free & free & free \\
\hline
\end{tabular}

Table 2

Best fit $\chi^{2}$ and upper limits on $\sum m_{\nu, \max }$ for the three different priors.

\begin{tabular}{lcc}
\hline prior type & best fit $\chi^{2}$ & $\sum m_{\nu, \max }(\mathrm{eV})(95 \%)$ \\
\hline $\mathrm{CMB}+\mathrm{LSS}$ & 24.81 & 2.96 \\
$\mathrm{CMB}+\mathrm{LSS}+\mathrm{BBN}+H_{0}$ & 25.66 & 2.65 \\
$\mathrm{CMB}+\mathrm{LSS}+\mathrm{BBN}+H_{0}+\mathrm{SNIa}$ & 25.71 & 2.47 \\
\hline
\end{tabular}

combination of CMB data with the PSCz data a bound of $N_{\nu}=6_{4.5}^{8}(95 \%)$ has been obtained 442] (see also Table 3). Interestingly, this has provided the first detection of the cosmic neutrino background around a redshift of 1000, and the first measurement independent of big bang nucleosynthesis.

It should also be noted that the flavour dependence of the BBN bound relies on the assumption that there is no mixing between different flavours during BBN. However, if the solar LMA solution turns out to be correct it is almost inevitable that there is significant equilibration between $\nu_{e}$ and $\nu_{\mu}$ prior to BBN. It has been shown that this significantly strengthens the bound because a large $\nu_{\nu}$ or $\nu_{\tau}$ lepton number will leak into the electron sector before BBN 50 54.

\section{Discussion}

Cosmology offers an interesting probe of neutrino physics which is complementary to terrestrial experiments.

Observations of the large scale structure power spectrum has already given bounds on the ab- solute value of neutrino masses which are comparable to, or stronger than the present bound from tritium endpoint measurements. For the present data an upper limit to the neutrino mass of $\sum m_{\nu} \lesssim 2.5-3 \mathrm{eV}$ can be derived. This can be compared to the present bound from the Mainz experiment of $m_{\nu_{e}}=\sum_{i}\left(\left|U_{e i}\right|^{2} m_{i}^{2}\right)^{1 / 2}=2.2 \mathrm{eV}$.

However, it should be noted that an even stronger upper bound can be put on neutrino masses if they are Majorana particles. In that case neutrinoless double beta decay is possible because lepton number is not a conserved quantity. The non-observation of such events has led to the bound

$m_{e e}=\sum_{j} U_{e j}^{2} m_{\nu_{j}}<0.27 \mathrm{eV}$,

where $U$ is the neutrino mixing matrix [65]. From observations of neutrinoless double beta decay it has indeed been claimed that positive evidence for non-zero neutrino masses has been obtained, with a favoured value in the range $0.11-0.56 \mathrm{eV}$ 66]. However, this claim is highly controversial and has been refuted by a number of other authors 67. At present it therefore seems safest 


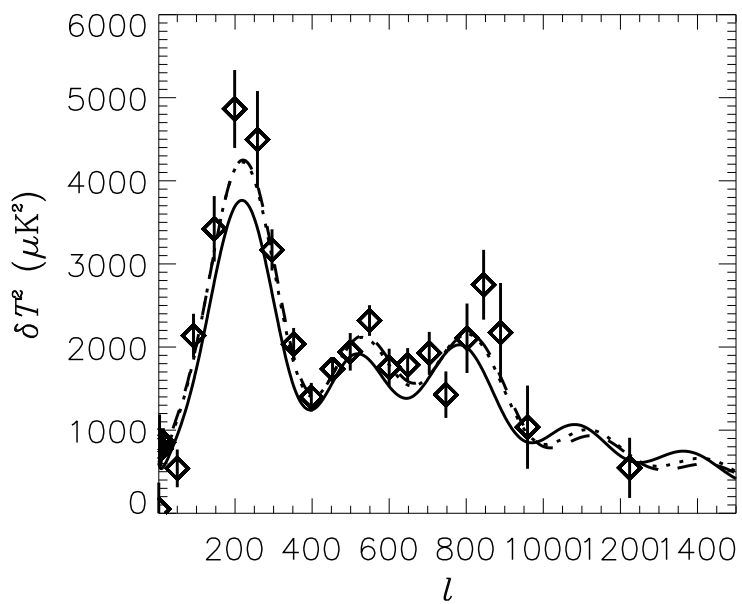

Figure 3. CMB power spectra for the best fits to the cosmological data. The full line is for $N_{\nu}=0$, the dashed for $N_{\nu}=7$, and the dotted for $N_{\nu}=$ 14. The data points are from the compilation by Wang, Tegmark and Zaldarriaga [36].

to regard neutrinoless double beta decay experiments as yielding only an upper limit on $N_{\nu}$.

In the coming years the large scale structure power spectrum will be measured even more accurately by the Sloan Digital Sky Survey, and at the same time the CMB anisotropy will be probed to great precision by the MAP and Planck satellites. By combining these measurements it was estimated by $\mathrm{Hu}$, Eisenstein and Tegmark that a sensitivity of about $0.3 \mathrm{eV}$ could be reached [28]. Currently an upgrade of the Mainz experiment by an order of magnitude, the KATRIN experiment, is planned. Such an experiment should take the limit on the electron neutrino mass down to about $0.2 \mathrm{eV}$. The prospects for measuring a neutrino mass of the order $0.1 \mathrm{eV}$, as suggested by oscillation experiments is therefore almost within reach.

Another cosmological probe of the neutrino mass is the so-called Z-burst scenario for ultrahigh energy cosmic rays 60, 61]. Neutrinos are not subject to the GZK cut-off which applies to protons [58,59]. Therefore it is in principle pos- sible that the primary particles for super GZK cosmic rays are neutrinos. One possibility which has been explored is that the neutrino-nucleon cross-section increases drastically at high CM energies, for instance due to the presence of large extra dimensions. The other possibility is that neutrinos have rest mass in the $\mathrm{eV}$ range. In that case high energy neutrinos can annihilate on cosmic background neutrinos with a large cross section if the CM energy is close to the Z-resonance, corresponding to a primary neutrino energy of $E_{\nu} \simeq 4 \times 10^{21} \mathrm{~m}_{\mathrm{eV}}^{-1} \mathrm{eV}$. This annihilation would produce high energy protons which could then act as primaries for the observed high energy cosmic rays. The observed ultrahigh energy cosmic ray flux can be explained if the heaviest neutrino has a mass larger than $\sim 0.1 \mathrm{eV}$. Therefore, if the Z-burst scenario turns out to be correct, it is in principle possible to measure a neutrino mass in this range 62 64.

With regards to the neutrino relativistic energy density, the present bound from BBN is roughly $N_{\nu} \lesssim 3.5-4$. The prospects for improving this bound in the future do not seem too bright. The present bound is most likely dominated by systematic effects in the measurement of helium abundances. BBN is also a very powerful probe of a possible neutrino lepton asymmetry, particularly if, as is indicated by data from SNO and Super-Kamiokande [3.4], the LMA mixing solution is correct. In this case, an upper bound on the lepton asymmetry for any flavour is roughly $\mu_{\nu_{i}} / T \lesssim 0.07$ [52].

The combination of CMB and large scale structure data can also be used for constraining the neutrino relativistic energy density. At present the CMB data are not of sufficient accuracy to yield a bound which is competitive with that from BBN, the present bound being $N_{\nu}=6_{4.5}^{8}(95 \%)$ (see also Table 3). However, this bound applies to a very different epoch and therefore puts significant constraints on possible entropy production after BBN but before CMB formation.

In the near future a much more accurate determination of $N_{\nu}$ from CMB measurements will become possible thanks to the satellites MAP and Planck. It was estimated by Lopez et al. [55] that it would be possible to measure $\Delta N_{\nu} \sim 0.04$ us- 
Table 3

Best fit values and $2 \sigma(95 \%)$ limits on $N_{\nu}$ for different priors and two different data sets. Both priors and data sets are discussed in Ref. [42]

\begin{tabular}{llc}
\hline prior type & WTZ & COBE+Boomerang \\
\hline CMB only & $8_{-8}^{+11}$ & $7_{-7}^{+17}$ \\
CMB + BBN $+H_{0}$ & $8_{-7}^{+9.5}$ & $4_{-4}^{+13}$ \\
CMB + BBN $+H_{0}+$ LSS & $6_{-4.5}^{+8}$ & $9_{-6.5}^{+8}$ \\
\hline
\end{tabular}

ing Planck data. However, this is probably overly optimistic and a more reasonable estimate seems to be $\Delta N_{\nu} \sim 0.1-0.2[56$.

This will also allow for a possible detection of sterile neutrinos mixing with ordinary neutrinos in the early universe over a wide range of parameter space [57.

\section{REFERENCES}

1. S. Fukuda et al. [Super-Kamiokande Collaboration], Phys. Rev. Lett. 85, 3999 (2000) arXiv:hep-ex/0009001.

2. N. Fornengo, M. C. Gonzalez-Garcia and J. W. Valle, Nucl. Phys. B 580 (2000) 58 arXiv:hep-ph/0002147.

3. See the SNO homepage http://sno.phy.queensu.ca

4. J. N. Bahcall, M. C. Gonzalez-Garcia and C. Pena-Garay, arXiv:hep-ph/0204314.

5. V. A. Kostelecky and S. Samuel, Phys. Lett. B 318, 127 (1993).

6. G. M. Fuller, J. R. Primack and Y. Z. Qian, Phys. Rev. D 52, 1288 (1995) arXiv:astro ph/9502081.

7. D. O. Caldwell and R. N. Mohapatra, Phys. Lett. B 354, 371 (1995) arXiv:hep$\mathrm{ph} / 9503316$.

8. S. M. Bilenky, C. Giunti, C. W. Kim and S. T. Petcov, Phys. Rev. D 54, 4432 (1996) arXiv:hep-ph/9604364.

9. S. F. King and N. N. Singh, Nucl. Phys. B 596, 81 (2001) arXiv:hep-ph/0007243.

10. H. J. He, D. A. Dicus and J. N. Ng, arXiv:hepph/0203237.

11. A. Ioannisian and J. W. Valle, Phys. Lett. B 332, 93 (1994) arXiv:hep-ph/9402333.

12. P. Bamert and C. P. Burgess, Phys. Lett. B
329, 289 (1994) arXiv:hep-ph/9402229.

13. R. N. Mohapatra and S. Nussinov, Phys. Lett. B 346, 75 (1995) arXiv:hep-ph/9411274.

14. H. Minakata and O. Yasuda, Phys. Rev. D 56, 1692 (1997) arXiv:hep-ph/9609276.

15. F. Vissani, arXiv:hep-ph/9708483.

16. H. Minakata and O. Yasuda, Nucl. Phys. B 523, 597 (1998) arXiv:hep-ph/9712291.

17. J. R. Ellis and S. Lola, Phys. Lett. B 458, 310 (1999) arXiv:hep-ph/9904279.

18. J. A. Casas, J. R. Espinosa, A. Ibarra and I. Navarro, Nucl. Phys. B 556, 3 (1999) arXiv:hep-ph/9904395.

19. J. A. Casas, J. R. Espinosa, A. Ibarra and I. Navarro, Nucl. Phys. B 569, 82 (2000) arXiv:hep-ph/9905381.

20. E. Ma, J. Phys. G 25, L97 (1999) arXiv:hep$\mathrm{ph} / 9907400$.

21. R. Adhikari, E. Ma and G. Rajasekaran, Phys. Lett. B 486, 134 (2000) arXiv:hepph/0004197.

22. J. Bonn et al., Nucl. Phys. Proc. Suppl. 91, 273 (2001).

23. S. Hannestad and J. Madsen, Phys. Rev. D 52, 1764 (1995) arXiv:astro-ph/9506015.

24. A. D. Dolgov, S. H. Hansen and D. V. Semikoz, Nucl. Phys. B 503, 426 (1997) arXiv:hep-ph/9703315.

25. G. Mangano, G. Miele, S. Pastor and M. Peloso, arXiv:astro-ph/0111408.

26. A. G. Doroshkevich, Y. B. Zeldovich and R. A. Sunyaev, Sov. Astron. Lett. 6, 252 (1980)

27. A. G. Doroshkevich, M. Y. Khlopov, R. A. Sunyaev, Y. B. Zeldovich and A. S. Szalay, In *Baltimore 1980, Proceedings, Relativistic Astrophysics*, 32-42.

28. W. Hu, D. J. Eisenstein and M. Tegmark, 
Phys. Rev. Lett. 80, 5255 (1998) arXiv:astro$\mathrm{ph} / 9712057$.

29. R. A. Croft, W. Hu and R. Dave, Phys. Rev. Lett. 83, 1092 (1999) arXiv:astro$\mathrm{ph} / 9903335$.

30. M. Fukugita, G. C. Liu and N. Sugiyama, Phys. Rev. Lett. 84, 1082 (2000) arXiv:hep ph/9908450.

31. O. Elgaroy et al., arXiv:astro-ph/0204152.

32. A. Lewis and S. Bridle, arXiv:astro$\mathrm{ph} / 0205436$.

33. S. Hannestad, arXiv:astro-ph/0205223.

34. S. Burles, K. M. Nollett and M. S. Turner, arXiv:astro-ph/0010171.

35. S. Hannestad, Phys. Rev. D 61, 023002 (2000).

36. X. Wang, M. Tegmark and M. Zaldarriaga, arXiv:astro-ph/0105091 (WTZ).

37. A. J. S. Hamilton and M. Tegmark, Mon. Not. R. Astron. Soc. 330, 506 (2002).

38. K. N. Abazajian, arXiv:astro-ph/0205238.

39. E. Lisi, S. Sarkar and F. L. Villante, Phys. Rev. D 59 (1999) 123520 arXiv:hep$\mathrm{ph} / 9901404$.

40. H. S. Kang and G. Steigman, Nucl. Phys. B 372 (1992) 494.

41. S. Hannestad, Phys. Rev. Lett. 85 (2000) 4203 arXiv:astro-ph/0005018.

42. S. Hannestad, Phys. Rev. D 64 (2001) 083002 arXiv:astro-ph/0105220.

43. S. Esposito, G. Mangano, A. Melchiorri, G. Miele and O. Pisanti, Phys. Rev. D 63 (2001) 043004 arXiv:astro-ph/0007419.

44. J. P. Kneller, R. J. Scherrer, G. Steigman and T. P. Walker, Phys. Rev. D 64 (2001) 123506 arXiv:astro-ph/0101386.

45. C. B. Netterfield et al. [Boomerang Collaboration], arXiv:astro-ph/0104460.

46. A. T. Lee et al., astro-ph/0104459.

47. S. Padin et al., Astrophys. J. Lett. 549 (2001) L1.

48. N. W. Halverson et al., arXiv:astro$\mathrm{ph} / 0104489$.

49. S. H. Hansen, G. Mangano, A. Melchiorri, G. Miele and O. Pisanti, arXiv:astro$\mathrm{ph} / 0105385$.

50. C. Lunardini and A. Y. Smirnov, Phys. Rev. D 64, 073006 (2001) arXiv:hep-ph/0012056.
51. S. Pastor, G. G. Raffelt and D. V. Semikoz, arXiv:hep-ph/0109035.

52. A. D. Dolgov, S. H. Hansen, S. Pastor, S. T. Petcov, G. G. Raffelt and D. V. Semikoz, Nucl. Phys. B 632, 363 (2002) arXiv:hep$\mathrm{ph} / 0201287$.

53. K. N. Abazajian, J. F. Beacom and N. F. Bell, Phys. Rev. D 66, 013008 (2002) arXiv:astro$\mathrm{ph} / 0203442$.

54. Y. Y. Wong, Phys. Rev. D 66, 025015 (2002) arXiv:hep-ph/0203180.

55. R. E. Lopez, S. Dodelson, A. Heckler and M. S. Turner, Phys. Rev. Lett. 82, 3952 (1999) arXiv:astro-ph/9803095.

56. R. Bowen et al., arXiv:astro-ph/0110636.

57. S. Hannestad and G. Raffelt, Phys. Rev. D 59 (1999) 043001 arXiv:astro-ph/9805223.

58. K. Greisen, Phys. Rev. Lett. 16 (1966) 748.

59. G. T. Zatsepin and V. A. Kuzmin, JETP Lett. 4 (1966) 78 [Pisma Zh. Eksp. Teor. Fiz. 4 (1966) 114].

60. T. J. Weiler, cut-off," Astropart. Phys. 11 (1999) 303 arXiv:hep-ph/9710431.

61. D. Fargion, B. Mele and A. Salis, rays," Astrophys. J. 517 (1999) 725 arXiv:astro$\mathrm{ph} / 9710029$.

62. H. Pas and T. J. Weiler, Phys. Rev. D 63 (2001) 113015 arXiv:hep-ph/0101091.

63. Z. Fodor, S. D. Katz and A. Ringwald, arXiv:hep-ph/0105064.

64. A. Ringwald, arXiv:hep-ph/0111112.

65. H. V. Klapdor-Kleingrothaus et al., arXiv:hep-ph/0103062.

66. H. V. Klapdor-Kleingrothaus, A. Dietz, H. L. Harney and I. V. Krivosheina, Mod. Phys. Lett. A 16, 2409 (2001) arXiv:hep$\mathrm{ph} / 0201231$.

67. C. E. Aalseth et al., Mod. Phys. Lett. A 17, 1475 (2002) arXiv:hep-ex/0202018. 